\title{
Anti-SLA and AMA-positive celiac disease: A report of two cases and evaluation of autoimmune liver serology
}

\author{
Nurhan Demir ${ }^{1}$, (i) Cumali Efe ${ }^{1}$, (i) Seyhmus Olmez ${ }^{2}$, (i) Arzu Onur ${ }^{3}$, (i) Eric M. Yoshida ${ }^{4}$ \\ ${ }^{1}$ Department of Gastroenterology, Diyarbakir Health Science University Training and Research Hospital, Diyarbakir, Turkey; ${ }^{2}$ Department of Gastroenterology, \\ Adana Numune Training and Research Hospital, Adana, Turkey; ${ }^{3}$ Department of Microbiology, Diyarbakir Health Science University Training and Research \\ Hospital, Diyarbakir, Turkey; ${ }^{4}$ Division of Gastroenterology, University of British Columbia, Vancouver, Canada
}

\begin{abstract}
Background and Aim: Antibodies to soluble liver antigen (anti-SLA) and anti-mitochondrial autoantibodies (AMA) are two specific serological markers of autoimmune hepatitis $(\mathrm{AIH})$ and primary biliary cholangitis (PBC). They are rarely detected in the sera of patients with non-liver autoimmune disorders.

Materials and Methods: The biochemical, serological, and histological findings of two celiac disease (CD) patients who were seropositive for antiSLA and/or AMA were evaluated.

Results: The data of two female patients who were 28 and 34 years old at the time of CD diagnosis were analyzed. The first patient presented with elevated liver function tests (LFTs) and immunoglobulin (Ig) G values. She was seropositive for both anti-SLA and AMA. A liver biopsy suggested features of AIH but no bile duct injury was noted. In addition to a gluten-free diet (GFD), immunosuppressive therapy was administered to normalize the LFTs. The second patient presented with elevated LFTs, a high IgG level, and a positive anti-SLA finding. A GFD was initiated, which resulted in an excellent clinical and biochemical response. Seropositivity for AMA in the first patient and anti-SLA in the second patient remained unchanged during follow-up but neither patient developed primary biliary cholangitis or AIH.
\end{abstract}

Conclusion: Despite the high specificity of anti-SLA and AMA, these autoantibodies can be detected in $\mathrm{CD}$ without having any clinical relevance.

Keywords: Antibodies to soluble liver antigen; anti-mitochondrial autoantibodies; autoimmune hepatitis; celiac disease; primary biliary cholangitis.

\section{Introduction}

Autoantibodies have an important role in the diagnosis and classification of autoimmune hepatitis (AIH). Type $1 \mathrm{AIH}$ is associated with antinuclear antibodies (ANA) and/or smooth muscle antibodies (SMA), while type $2 \mathrm{AIH}$ includes antibodies to the liver/kidney microsome (anti-LKM-1) and/or liver cytosolic protein type $1 .{ }^{[1-4]}$ However, these autoantibodies are not fully disease-specific, as they are detected in various forms of chronic liver disorders, other immune-mediated diseases, and even in healthy individuals. Conversely, a finding of antibodies to

Received: February 13, 2020; Accepted: April 11, 2020; Available online: May 21, 2020

Corresponding author: Cumali Efe; Diyarbakir Saglik Bilimleri Universitesi Egitim ve Arastirma Hastanesi, Gastroenteroloji Klinigi, Diyarbakir, Turkey

Phone: +90 50550255 96; e-mail: drcumi21@hotmail.com

(c) (i) (5) OPEN ACCESS

DPEN ACCESS (C) Copyright 2020 by Hepatology Forum - Available online at www.hepatologyforum.org soluble liver antigen (anti-SLA) has been suggested as a highly specific marker for AIH. ${ }^{[5-7]}$

Anti-mitochondrial autoantibodies (AMA) are considered the serological hallmark of primary biliary cholangitis (PBC). Due to the high sensitivity and specificity of AMA, a liver biopsy is not necessary for the diagnosis of PBC when AMA and a cholestatic biochemical profile are present. $^{[8,9]}$

Celiac disease (CD) is a common, chronic, immune-mediated disorder of the small intestine. $\mathrm{CD}$ is induced by dietary wheat, barley, and rye. ${ }^{[10]}$ $\mathrm{CD}$ is not restricted to the small intestine; it has been recognized to be a multisystem disorder that may affect other organs, such as the nervous system, bones, skin, and the liver. CD may first present with features of liver injury. ${ }^{[1]}$ Seropositivity for autoimmune liver serology is seen in patients with $\mathrm{CD}$ and some patients are diagnosed with co-existing $\mathrm{AIH}$, $\mathrm{PBC}$, or primary sclerosing cholangitis. ${ }^{[10,11]}$ Gastroenterologists need to consider $\mathrm{CD}$ in the differential diagnosis of abnormal liver tests and be aware of associated autoimmune liver disorders. In this study, two cases of CD associated with AMA and anti-SLA, both highly specific markers for PBC and $\mathrm{AIH}$ are presented. Diagnostic pitfalls for CD patients who have autoimmune liver serology are also discussed. Our findings may help gastroenterologists better understand the diagnostic complexity of $\mathrm{CD}$ and associated or coexisting autoimmune liver diseases.

\section{Materials and Methods}

The study was performed using medical data from Hacettepe University and Gazi Yaşargil Education and Research Hospital. The diagnosis of AIH was made according to the simplified criteria suggested by the International Autoimmune Hepatitis Group. ${ }^{[12]}$ Treatment of AIH was assessed according to the American Association for the Study of Liver Disease guidelines. Normalization of serum aminotransferases and immunoglobulin (Ig) $\mathrm{G}$ levels has been defined as a complete biochemical response.$^{[1]} \mathrm{CD}$ was diagnosed based on serology and duodenal biopsy findings while patients had a diet that contained gluten. ${ }^{[13]}$ The ethics committee of Diyarbakır Gazi Yaşargil Education and Research Hospital approved this study.

\section{Serological Assessment}

ANA, SMA, LKM-1, anti-SLA, and AMA with the M2 fraction were assessed using an immunoblotting technique. A titer of 1/40 or above for AMA-M2 was considered positive when the indirect immunofluorescence technique was applied. A commercially available enzymelinked immunosorbent assay (ELISA) was used to detect IgA and IgG 
Table 1. Biochemical, serological, treatment responses, and outcomes of the patients

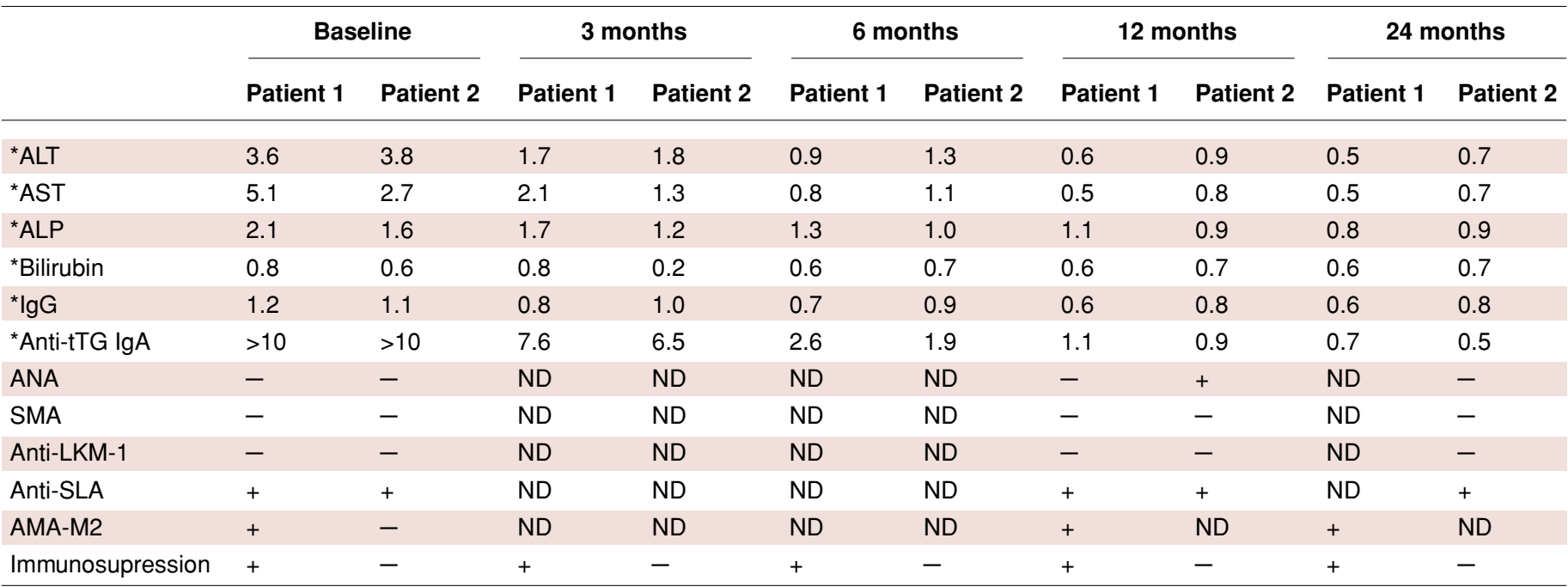

*Expressed as xUNL (upper normal limit). ALP: Alkaline phosphatase; ALT: Alanine aminotransferase; AMA-M2: Anti-mitochondrial M2 antibody; ANA: Antinuclear

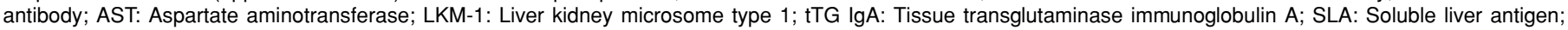
SMA: Smooth muscle antibody.

anti-tissue transglutaminase (tTG) antibody levels. To detect anti-SLA, the commercially available Euroline liver profile kit (Euroimmun, Lubeck, Germany) was used. The test was performed using a recombinant SLA antigen. This method detects the molecular weight of 50 $\mathrm{kDa}$ of SLA protein, which is involved in the regulation of the UGA suppressor tRNA-associated protein. ${ }^{[14]}$

\section{Histological Evaluation}

A local pathologist reviewed the liver tissue and duodenum specimens. The typical histology for AIH was defined in terms of the presence of interface hepatitis, lymphocytic/lymphoplasmacytic infiltrates in the portal tracts and extending into the lobule, and hepatic rosette formation. Subtotal villous atrophy and an intraepithelial lymphocyte count of $>35 / 100$ enterocytes were required for a diagnosis of CD.

\section{Results}

Two patients who were 28 and 34 years old at the time of diagnosis were included in the study. The general characteristics and outcomes of the patients are presented in Table 1. Neither patient had an alcohol or drug history, serology for viral hepatitis (anti-HAV IgM, HBsAg, anti-HBc IgM, anti-HCV), drug-induced liver injury (DILI), or any other cause of hepatitis, such as Wilson's disease or hemochromatosis. A GFD was the initial step for both patients. Patient \#1 showed no improvement in LFTs with the GFD, and immunosuppression was administered. Patient \#2 demonstrated biochemical remission in LFTs with a GFD and received no immunosuppression. AMA continued to be seropositive in the following two serological work-ups of patient \#1. Anti-SLA positivity remained unchanged in the patients through multiple evaluations; one patient was tested two times and the other three times.

\section{Case 1}

A 34-year-old woman presented with a history of malaise, diarrhea, and weight loss. Her weight and height were $43.3 \mathrm{~kg}$ and $161 \mathrm{~cm}$, respectively. The initial laboratory evaluation was consistent with iron deficiency anemia: hemoglobin $10.5 \mathrm{~g} / \mathrm{dL}(11-16 \mathrm{~g} / \mathrm{dL})$, total iron binding capacity $489 \mathrm{mg} / \mathrm{dL}(250-425 \mathrm{mg} / \mathrm{dL})$, and ferritin 5.3 $\mathrm{ng} / \mathrm{mL}$ (normal: $28-365 \mathrm{ng} / \mathrm{mL}$ ). Other values were alanine aminotransferase (ALT) $197 \mathrm{U} / \mathrm{L}(0-54 \mathrm{U} / \mathrm{L})$, aspartate aminotransferase (AST) $175 \mathrm{U} / \mathrm{L}(0-34 \mathrm{U} / \mathrm{L})$, alkaline phosphatase (ALP) $314 \mathrm{U} / \mathrm{L}$ (40-150 U/L), gamma-glutamyl transferase (GGT) $75 \mathrm{U} / \mathrm{L}$ (5-64 $\mathrm{U} / \mathrm{L})$, total bilirubin $0.9 \mathrm{mg} / \mathrm{dL}(0.6-1.2 \mathrm{mg} / \mathrm{dL})$, total protein $68 \mathrm{~g} /$ $\mathrm{dL}(64-83 \mathrm{~g} / \mathrm{dL})$, and albumin $39 \mathrm{~g} / \mathrm{dL}(35-45 \mathrm{~g} / \mathrm{dL})$. The serum IgG level was $18.6 \mathrm{~g} / \mathrm{L}$ (7-16.1 g/L). Anti-tTG IgA and IgG were strongly positive $(>200 \mathrm{U} / \mathrm{mL})$. In the liver immune serology, only AMA-M2 $(++)$ and anti-SLA $(++)$ were positive at titers of $1 / 160$ and $63 \mathrm{RU} /$ $\mathrm{mL}$, respectively. Bone mineral density (BMD) disclosed severe osteoporosis, a T-score of $-3.3 \mathrm{SD}$ at the lumbar spine and -2.1 SD at the femur neck. Magnetic resonance cholangiography results were normal. Endoscopy disclosed a mosaic pattern and flattening in the duodenal folds. Examination of duodenal biopsies showed subtotal villous atrophy, lymphocyte infiltration within the subepithelium, and increased intraepithelial lymphocytes, consistent with a Marsh grade 3b. Vitamin D3 $1200 \mathrm{mcg} /$ day, calcium $800 \mathrm{mg} /$ day, and alendronate $70 \mathrm{mg} /$ week were initiated as well as a GFD. Her clinical symptoms (diarrhea, depressive mood, and general) were significantly improved within 6 weeks, and the levels of ALT, AST, and IgG increased to $403 \mathrm{U} / \mathrm{L}, 276 \mathrm{U} / \mathrm{L}$, and $29.1 \mathrm{~g} / \mathrm{L}$, respectively. A liver biopsy revealed periportal fibrosis, lymphoplasmocytic infiltration, and moderate to severe interface hepatitis, but no features of bile duct injury. Prednisolone $40 \mathrm{mg} /$ day and azathioprine $50 \mathrm{mg}$ /day were added to the treatment. The LFTs normalized in the fifth month of therapy. The patient was in complete biochemical remission with prednisolone 2.5 $\mathrm{mg} /$ day and azathioprine $100 \mathrm{mg}$ /day at her last follow-up.

\section{Case 2}

A 28-year-old woman was evaluated for an unclear elevation of liver aminotransferases. Her medical history was characterized by growth failure, long-term treatment for iron deficiency anemia, and hypothy- 


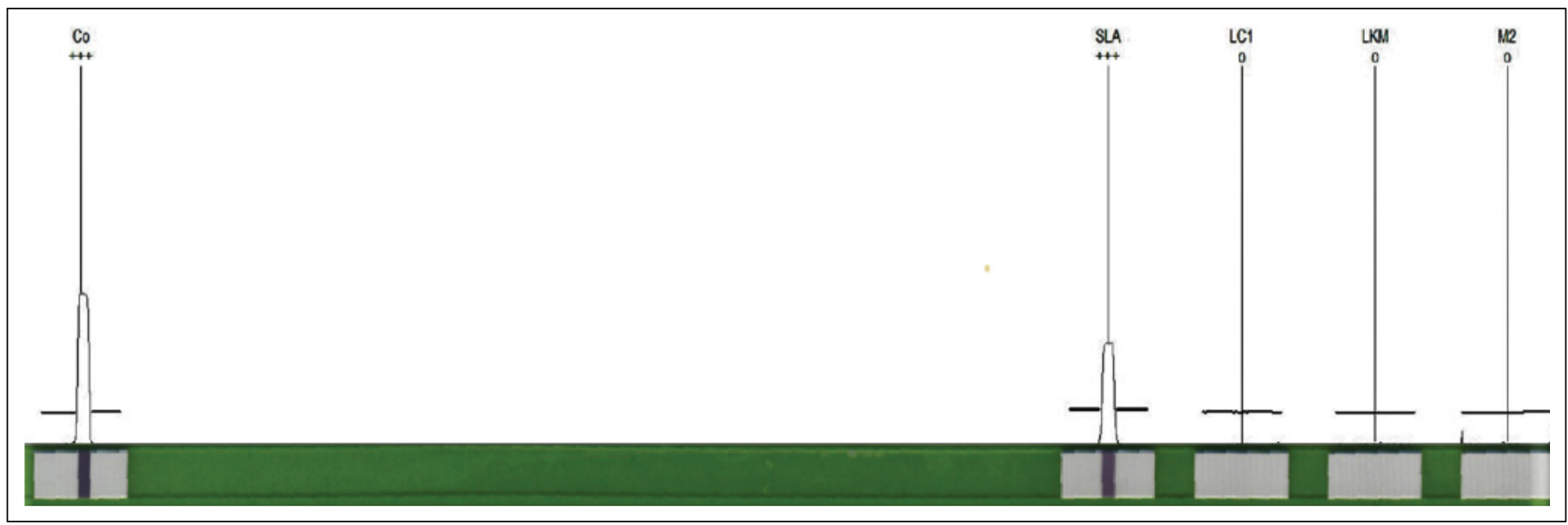

Figure 1. Strong color reaction is visible on the SLA band of patient \#2.

Co: Cobalt; LC1: Liver cytosolic protein type 1; LKM: Liver-kidney microsome; M2: Anti-mitochondrial antibody type 2; SLA: Soluble liver antigen.

roidism. Her weight and height were $40.5 \mathrm{~kg}$ and $155 \mathrm{~cm}$, respectively. The initial laboratory evaluation revealed iron deficiency anemia: hemoglobin $10.4 \mathrm{~g} / \mathrm{dL}(11-16 \mathrm{~g} / \mathrm{dL})$, total iron binding capacity $492 \mathrm{mg} /$ $\mathrm{dL}(250-425 \mathrm{mg} / \mathrm{dL})$, ferritin $15.3 \mathrm{ng} / \mathrm{mL}$ (normal: $28-365 \mathrm{ng} / \mathrm{mL}$ ), and ALT $129 \mathrm{U} / \mathrm{L}$ (0-34 U/L), AST 94 U/L (0-35 U/L), ALP $196 \mathrm{U} / \mathrm{L}$ (40-150 U/L), GGT $75 \mathrm{U} / \mathrm{L}$ (5-64 U/L), total bilirubin $0.8 \mathrm{mg} / \mathrm{dL}$ $(0.4-1.2 \mathrm{mg} / \mathrm{dL})$, total protein $63 \mathrm{~g} / \mathrm{dL}(64-83 \mathrm{~g} / \mathrm{dL})$, albumin $36 \mathrm{~g} / \mathrm{dL}$ $(35-45 \mathrm{~g} / \mathrm{dL})$, and IgG $17.3 \mathrm{~g} / \mathrm{L}(6.9-15.4 \mathrm{~g} / \mathrm{L})$. The CD markers of anti-tTG IgA and IgG were strongly positive $(>200 \mathrm{U} / \mathrm{mL})$. ANA, SMA, and anti-LKM-1 were negative, only anti-SLA was positive $(+++)$ at a titer of $83 \mathrm{RU} / \mathrm{mL}$ (Fig. 1). BMD measurement disclosed severe osteoporosis, with a T-score of $-4.3 \mathrm{SD}$ at the lumbar spine and -2.8 SD at the femur neck. Endoscopy revealed a mosaic pattern and flattening of duodenal folds. A duodenal biopsy examination was consistent with Marsh grade 3b CD. Vitamin D3 $1200 \mathrm{mcg} /$ day, calcium $800 \mathrm{mg} /$ day, and alendronate $70 \mathrm{mg} /$ week and a GFD were initiated. After 4 weeks, follow-up indicated that the CD symptoms were significantly improved and that the serum ALT and AST levels had decreased to $107 \mathrm{U} / \mathrm{L}$ and $80 \mathrm{U} / \mathrm{L}$, respectively. These values normalized after 12 months of follow-up. The LFTs and IgG levels remained normal during follow-up without immunosuppressive therapy.

\section{Discussion}

Elevated liver transaminases are observed in $40-50 \%$ of untreated CD cases, and CD may be the cause of unexplained hypertransaminasemia in about $10 \%$ of patients. ${ }^{[10]}$ CD-related liver injury and autoimmune liver disease are two main causes of a liver enzyme abnormality in the context of CD. ${ }^{[11]}$ A possible pathogenetic mechanism for hypertransaminasemia in $\mathrm{CD}$ may be impaired gut mucosal integrity, resulting in increased mucosal permeability that triggers autoantibody (anti-tTG) or toxin-mediated liver injury. LFTs usually normalize with a GFD in $\mathrm{CD}$, but specific treatment is necessary in patients with autoimmune liver diseases. ${ }^{[10,11]}$ Our patients had serum transaminases $<5$ UNL at the time of presentation. Therefore, we initially managed both patients with a GFD alone. Patient \#1 did not show improvement in the LFTs after implementing a GFD. It was considered a case of CD with AIH, and the addition of immunosuppressive treatment led to biochemical remission. Patient \#2 had normalized LFTs with a GFD and it was considered CD with an associated liver injury.
A close association between $\mathrm{CD}$ and autoimmune-mediated liver disease (AILD) has been well described in large, cohort-based studies. Approximately, 3-7\% of PBC patients and 3-6\% with AIH have concomitant CD. ${ }^{[15]}$ Patients with CD may have seropositivity for AILD serology. Carroccio et al. ${ }^{[16]}$ reported that $24 \%$ of $\mathrm{CD}$ patients had a median 1/80 ANA positivity (range, 1/40:1/1280). SMA, AMA, and LKM-1 have also been detected in the sera of patients with CD. ${ }^{[17]}$

Antibodies to SLA have been suggested as disease-specific autoantibodies and therefore to have high diagnostic value in AIH. Anti-SLA antibodies were first described by Manns et al. ${ }^{[18]}$ in the sera of 23 patients with chronic active hepatitis. In later studies, the frequency of anti-SLA has been reported as $6-58 \%$ in AIH. ${ }^{[5]}$ They are rarely detected in other disorders or in healthy populations. In a study of 3026 patients, anti-SLA was detected only in AIH and overlap patients. ${ }^{[6,14]}$ In a French study, ${ }^{[19]} 72(89 \%)$ of 81 anti-SLA-positive patients had AIH or variant diagnoses, while $3 \%$ had DILI. Subsequent reports have shown that some drugs are potential triggers for AIH and that indolent or asymptomatic AIH may become clinically evident after DILI. ${ }^{[20]}$ Therefore, the absence of detailed information about the clinical features and outcome of these patients makes it difficult to evaluate anti-SLA positivity with DILI. Anti-SLA positivity has been reported in a few patients with $\mathrm{HCV}$ and $\mathrm{PBC} \cdot{ }^{[7,21]}$ Here, we report a rare finding of anti-SLA positivity in patients with non-hepatic autoimmune disease.

Anti-SLA assessment techniques rarely give false positive results. In one study, some patients who were anti-SLA positive according to an ELISA were negative after confirmation testing with the blotting technique ${ }^{[6]}$ Importantly, all of these patients had weak or borderline reactivity in the ELISA assay. Our two patients were evaluated using the immunoblotting technique and showed very strong anti-SLA positivity. In serial measurements with the same technique, the anti-SLA status did not change.

The prognostic value of anti-SLA still remains controversial. Some studies have suggested that anti-SLA-positive AIH patients demonstrated a poor prognosis and more frequent relapses; however, two recent studies could not confirm this association. ${ }^{[6,7,22,23]}$ Both of our patients had favorable disease outcomes, including a patient managed only with a GFD.

In our study, patient \#1 had additional AMA-M2 seropositivity, which is highly specific for PBC. ${ }^{[8]}$ The serum ALP levels were also greater 
than $2 \mathrm{x}$ UNL in this patient. AMA may be detected in patients with liver diseases (AIH, HCV, acute liver failure), in non-liver autoimmune diseases (Sjögren syndrome, systemic sclerosis, rheumatoid arthritis), and even in healthy individuals. ${ }^{[8,9]}$ ALP is not only produced in the liver but also in bone, intestine, and leucocytes. Several bone diseases or malignancies may be the cause of high serum ALP levels. Interestingly, ALP may be artificially high in patients with high serum IgG levels. ${ }^{[24,25]} \mathrm{Pa}$ tient \#1 had osteoporosis and an elevated IgG level at first presentation. The high ALP level did not reflect liver origin and returned to normal levels with supportive treatment for osteoporosis and immunosuppressive therapy. The diagnosis of AIH/PBC overlap could be made in this patient based on the serological and laboratory findings. Importantly, the current diagnostic criteria strongly recommended a diagnosis of overlap syndrome for suggestive liver histology findings.

In conclusion, we have presented two cases of $\mathrm{CD}$ that presented with specific auto-antibodies (anti-SLA and AMA) for AIH and PBC. After careful evaluation, patient \#1 was diagnosed as CD-AIH with AMA-M2 positivity as an associated immunological phenomenon. Patient \#2 showed good clinical and biochemical response to a GFD and was considered $\mathrm{CD}$ with associated hepatitis, despite having highly specific autoantibodies to AIH. The association between $\mathrm{CD}$ and autoimmune liver diseases is a well-known clinical entity. We believe our report contributes to understanding the serological complexity of this relationship.

Informed Consent: Written informed consent was obtained from patients who participated in this study.

Peer-review: Externally peer-reviewed.

Author Contributions: Concept - ND, CE; Design - ND; Supervision - CE; Materials - SO, AO; Data Collection and/or Processing - ND; Analysis and/or Interpretation - SO, AO; Literature Search - ND, AO; Writing - ND, CE, EMY; Critical Reviews - CE, EMY.

Conflict of Interest: The authors have no conflict of interest to declare.

Financial Disclosure: The authors declared that this study has received no financial support.

\section{References}

1. Manns MP, Czaja AJ, Gorham JD, Krawitt EL, Mieli-Vergani G, Vergani D, et al; American Association for the Study of Liver Diseases. Diagnosis and management of autoimmune hepatitis. Hepatology 2010;51(6):2193-2213.

2. Johanet C, Beleoken E, Ballot E. Autoantibodies in autoimmune hepatitis: antinuclear antibodies (ANA). Clin Res Hepatol Gastroenterol 2012;36(4):394-396.

3. Johanet C, Ballot E. Auto-antibodies in autoimmune hepatitis: anti-smooth muscle antibodies (ASMA). Clin Res Hepatol Gastroenterol 2012;36(2):189-91

4. Johanet C, Ballot E. Autoantibodies in autoimmune hepatitis: anti-liver kidney microsome type 1 (anti-LKM1) and anti-liver cytosol type 1 (anti-LC1) antibodies. Clin Res Hepatol Gastroenterol 2013;37(2):216-218.

5. Johanet C, Ballot E. Auto-antibodies in autoimmune hepatitis: anti-soluble liver antigen (SLA). Clin Res Hepatol Gastroenterol 2012;36(3):244-246.

6. Baeres M, Herkel J, Czaja AJ, Wies I, Kanzler S, Cancado EL, et al. Establishment of standardised SLA/LP immunoassays: specificity for autoim- mune hepatitis, worldwide occurrence, and clinical characteristics. Gut 2002;51(2):259-264.

7. Efe C, Ozaslan E, Wahlin S, Purnak T, Muratori L, Quarneti C, et al. Antibodies to soluble liver antigen in patients with various liver diseases: a multicentre study. Liver Int 2013;33(2):190-196.

8. Chantran Y, Ballot É, Johanet C. Autoantibodies in primary biliary cirrhosis: antimitochondrial autoantibodies. Clin Res Hepatol Gastroenterol 2013;37(4):431-433.

9. Ozaslan E, Efe C, Gokbulut Ozaslan N. The diagnosis of antimitochondrial antibody-negative primary biliary cholangitis. Clin Res Hepatol Gastroenterol 2016;40(5):553-561.

10. Singh P, Arora A, Strand TA, Leffler DA, Catassi C, Green PH, et al. Global Prevalence of Celiac Disease: Systematic Review and Meta-analysis. Clin Gastroenterol Hepatol 2018;16(6):823-836.e2.

11. Rubio-Tapia A, Murray JA. The liver in celiac disease. Hepatology 2007;46(5):1650-1658

12. Hennes EM, Zeniya M, Czaja AJ, Parés A, Dalekos GN, Krawitt EL, et al; International Autoimmune Hepatitis Group. Simplified criteria for the diagnosis of autoimmune hepatitis. Hepatology 2008;48(1):169-176.

13. Ludvigsson JF, Bai JC, Biagi F, Card TR, Ciacci C, Ciclitira PJ, et al. Diagnosis and management of adult coeliac disease: guidelines from the British Society of Gastroenterology. Gut 2014;63(8):1210-1228.

14. Wies I, Brunner S, Henninger J, Herkel J, Kanzler S, Meyer zum Büschenfelde $\mathrm{KH}$, et al. Identification of target antigen for SLA/LP autoantibodies in autoimmune hepatitis. Lancet 2000;355(9214):1510-1515.

15. Volta U. Liver dysfunction in celiac disease. Minerva Med 2008;99(6):619629.

16. Carroccio A, D'Alcamo A, Cavataio F, Soresi M, Seidita A, Sciumè C, et al. High Proportions of People With Nonceliac Wheat Sensitivity Have Autoimmune Disease or Antinuclear Antibodies. Gastroenterology 2015;149(3):596-603.e1.

17. Caglar E, Ugurlu S, Ozenoglu A, Can G, Kadioglu P, Dobrucali A. Autoantibody frequency in celiac disease. Clinics (Sao Paulo) 2009;64(12):1195200.

18. Manns M, Gerken G, Kyriatsoulis A, Staritz M, Meyer zum Büschenfelde KH. Characterisation of a new subgroup of autoimmune chronic active hepatitis by autoantibodies against a soluble liver antigen. Lancet 1987;1(8528):292-294

19. Eyraud V, Chazouilleres O, Ballot E, Corpechot C, Poupon R, Johanet C. Significance of antibodies to soluble liver antigen/liver pancreas: a large French study. Liver Int 2009;29(6):857-864.

20. Weiler-Normann C, Schramm C. Drug induced liver injury and its relationship to autoimmune hepatitis. J Hepatol 2011;55(4):747-749.

21. Vitozzi S, Djilali-Saiah I, Lapierre P, Alvarez F. Anti-soluble liver antigen/ liver-pancreas (SLA/LP) antibodies in pediatric patients with autoimmune hepatitis. Autoimmunity 2002;35(8):485-492.

22. Ma Y, Okamoto M, Thomas MG, Bogdanos DP, Lopes AR, Portmann B, et al. Antibodies to conformational epitopes of soluble liver antigen define a severe form of autoimmune liver disease. Hepatology 2002;35(3):658-664.

23. Zachou K, Gampeta S, Gatselis NK, Oikonomou K, Goulis J, Manoussakis $\mathrm{MN}$, et al. Anti-SLA/LP alone or in combination with anti-Ro52 and fine specificity of anti-Ro52 antibodies in patients with autoimmune hepatitis. Liver Int 2015;35(2):660-672.

24. Poupon R. Primary biliary cirrhosis: a 2010 update. J Hepatol 2010;52(5):745-758.

25. Poupon R. Liver alkaline phosphatase: a missing link between choleresis and biliary inflammation. Hepatology 2015;61(6):2080-2090. 